\title{
Canova, le marbre et la montagne
}

Une poétique de la pierre dans les Lettres d'un voyageur

\section{Nicole Savy}

\section{(2) OpenEdition}

\section{Journals}

Édition électronique

URL : http://journals.openedition.org/recherchestravaux/228

DOI : 10.4000/recherchestravaux.228

ISSN : 1969-6434

Éditeur

UGA Éditions/Université Grenoble Alpes

Édition imprimée

Date de publication : 15 avril 2007

Pagination : 181-192

ISBN : 978-2-84310-107-7

ISSN : 0151-1874

Référence électronique

Nicole Savy, "Canova, le marbre et la montagne », Recherches \& Travaux [En ligne], 70 | 2007, mis en ligne le 04 mars 2009, consulté le 07 septembre 2020. URL : http://journals.openedition.org/ recherchestravaux/228; DOI : https://doi.org/10.4000/recherchestravaux.228 


\section{Canova, le marbre et la montagne Une poétique de la pierre dans les Lettres d'un voyageur}

Vénitien, historien de la photographie et guide d'une journée merveilleuse à Asolo et à Possagno.

L'une des nombreuses particularités des Lettres d'un voyageur, ce texte étrange, est la présence insistante dans un certain nombre de lettres, en plus de la première consacrée en grande partie au sculpteur Canova, du monde minéral et de la roche : non plus comme matériau pour l'artiste, ni seulement comme élément de décor naturel dans les paysages, mais comme constituant d'une poétique sandienne qui unit le corps, la connaissance et le rêve dans un rapport au monde profondément original.

\section{Canova, le marbre et la montagne}

Les rapports de George Sand avec la sculpture n'ont jamais donné lieu à une étude spécifique : d'abord parce qu'il est avéré que sa prédilection va à la peinture et au dessin, dont elle est grande amatrice et qu'elle pratique ellemême ; peut-être aussi parce que, la biographie contaminant la critique, la traversée météorique du sculpteur Clésinger dans la famille Sand n'aura laissé que des souvenirs douloureux.

C'est pourtant de sculpture que nous parle la première lettre. Antonio Canova (I757-1822), né à Possagno, dans la campagne de la Vénétie, fils d'artisan, apprit le métier de tailleur de pierre avant de devenir un sculpteur célèbre dans toute l'Europe, appelé par Napoléon Ir dont il fit le portrait, 
auteur de la belle statue de Pauline Bonaparte qui se trouve à la villa Borghese ou du groupe L'Amour et Psyché qu'on peut voir au musée du Louvre. Grand admirateur de Rome et de l'Antique, cet artiste néoclassique se caractérise par une grâce délicate et par une facture exceptionnellement habile. Que George Sand l'ait choisi n'est pas le seul fait des hasards touristiques : les marbres blancs et lisses de Canova sont, au milieu du XIX siècle, ce qu'on peut imaginer de plus allogène, quand on les rapporte aux roches brutes de la montagne dont ils sont issus.

La sculpture apparaît, au début des Lettres, comme la quintessence de l'art italien, le seul qui intéresse le destinataire de la première lettre, Alfred de Musset :

Tu te souviens que, quand nous partîmes de France, tu n'étais avide, disais-tu, que de marbres taillés. Tu m'appelais sauvage quand je te répondais que je laisserais tous les palais du monde pour aller voir une belle montagne de marbre brut dans les Apennins ou dans les Alpes. (Lettre I, p. 65I-652; p. 42 ${ }^{2}$.)

D'emblée est mis en place, à l'image des deux correspondants, un couple antinomique : la roche et la sculpture, la montagne et le palais, la nature et l'art. L'amoureux des arts va rapidement se lasser, d'après l'auteur de la lettre qui a pris résolument parti pour la nature, parce que non seulement celle-ci suscite l'admiration de l'esprit, mais encore elle procure du plaisir aux sens. Après avoir contemplé les statues de Canova, George Sand s'intéresse à leurs modèles qu'elle retrouve dans la région : des paysans au type Antique et surtout de belles filles à la lourde chevelure blonde, aux yeux couleur d'aigue marine, au teint de lys et de roses, dont le marbre est impuissant à rendre la transparence et les couleurs. Elle conclut encore à la supériorité de la nature 3 .

Et cela, malgré les pouvoirs démiurgiques du sculpteur, sur lesquels elle médite un instant :

Quand il avait coupé proprement un quartier de roche, savait-il que de cette main, formée aux rudes travaux, sortiraient tous les dieux de l'Olympe et tous les rois de la terre ? Pouvait-il deviner cette nouvelle race de souverains qui allait éclore et demander l'immortalité à son ciseau ? (Lettre I ; p. 675 ; p. 64.)

I. On ne résistera pas au plaisir d'en citer la description par Claude Simon : "Amour et Psyché je crois dans cette matière savonneuse blanche marbre de Carrare ou quoi elle à demi couchée une draperie cachant ses jambes le buste nu soulevé entourant de ses deux bras le cou de l'Amour penché sur ses lèvres et dans son dos des ailes de papillon en forme d'éventails [...] " La Bataille de Pharsale, Paris, Minuit, 1969, p. 46.

2. Selon la convention que nous adoptons pour ce volume: G. Sand, Lettres d'un voyageur, pages de l'édition de G. Lubin puis pages de l'édition de H. Bonnet.

3. On peut penser que quelques années plus tard, devenue familière de la symbolique maçonnique, Sand eût raisonné autrement : la pierre taillée symbolise un grade supérieur à celui de la pierre brute sur le chemin de la connaissance et de la lumière. 
Sauf qu'elle a déjà récusé l'immortalité de la sculpture, en citant curieusement un extrait de Jacques, le roman qu'elle n'a pas encore publié à la date de cette lettre. On y voit une statue de marbre blanc, immaculée au sortir des mains de son auteur, puis rongée par le temps, abandonnée à sa solitude, " misérable pierre " (lettre I ; p. 667 ; p. 57) noircie par la mousse, qui espère le gel qui va l'achever et la rendre à la poussière. Les statues aussi peuvent mourir et l'art retourner au néant. Elle y revient à la fin de la lettre en décrivant le temple de Canova "au beau marbre [...] déjà égrené par la gelée " (lettre I ; p. 676 ; p. 65), décrivant ainsi une boucle dans le paradigme du froid, froid des marbres néoclassiques rattrapé par le froid élémentaire de la nature meurtrière et victorieuse. D'ailleurs elle renvoie toute idée d'indestructibilité du minéral au rayon des mythes : la vieille maison prêtée au voyageur s'abîme, il tombe du gravier dans ses cheveux, les murs se fendent, les dalles gèlent ; " je m'inquiète de la destruction de la pierre et de la courte durée du ciment séculaire" (lettre VII ; p. 848 ; p. 228), déclare-t-elle quelques lettres plus loin.

Les Lettres d'un voyageur s'ouvrent donc sur le meurtre symbolique de l'art. La lettre II compare les « vieux piliers de marbre grec » à " des ossements desséchés » (p. 694; p. 82) ; la description de Torcello, l'une des îles de la lagune vénitienne, évoque des ruines : "Ça et là un chapiteau de marbre, un fragment de sculpture du Bas-Empire, une belle croix grecque brisée, percent dans les hautes herbes" (lettre III ; p. 734 ; p. I2I). La vie commence avec ces herbes, ou dans les canaux, au pied des vieux palais vénitiens, avec les mousses d'émeraude et les huîtres qui viennent s'y accrocher. Sand condamne les merveilles de Canova et de l'Antique : non comme l'Éternel de l'Exode interdisant qu'on taille les pierres au nom de leur caractère sacré ; mais d'un regard situé au-dessus de l'histoire humaine, à l'échelle géologique, au nom d'un naturalisme militant - au sens d'amour de la nature, celui des peintres de paysages de la période romantique. C'est la nature qui est la véritable artiste : le Voyageur admire dans le Bugey, pourtant moins beau que les Alpes, "une masse de roches tendres étrangement découpées, des bastions et des piliers que l'on croirait construits et sculptés par la main de l'homme " (lettre X; p. 893 ; p. 27I).

L'art se trouvant ainsi rejeté pour cause de mortalité, retournons donc à cette nature qui, elle, a le privilège d'avoir une histoire et sur laquelle l'écrivain se trouve dès lors obligé de reconstruire une esthétique autrement. 


\section{Le sentiment de la montagne}

Comme ses contemporains, George Sand, au moment où elle écrit ces Lettres, hérite d'une découverte et d'un goût tout juste centenaires, ce qu'on a appelé le sentiment de la montagne : on date de I740-1840 la conquête philosophique, esthétique et physique des Alpes du Mont-Blanc, d'abord par des Genevois comme Horace-Bénédict de Saussure, qui réussit après plusieurs tentatives l'ascension du mont Blanc en 1787 , et Marc-Théodore Bourrit ${ }^{4}$. Période où les naturalistes et les physiciens sont curieux de voir ce qu'on appelait alors les "glacières ", où de nombreux voyageurs rapportent des récits, descriptions pittoresques, cartes et guides, où dessinateurs et graveurs multiplient les vues des sommets, lacs et cascades, où les plus grands écrivains comme Rousseau, Chateaubriand et Hugo's liant altitude et élévation spirituelle, méditent face aux paysages les plus grandioses.

C'est dans ce mouvement qu'il faut situer Sand, fille spirituelle et littéraire, à ce titre comme à d'autres, de Jean-Jacques Rousseau. Murailles de granit, "pics terribles ", paysages désertiques caractérisent ces Alpes auxquelles l'auteur voue une admiration amère :

J'aime ces lieux incultes, inhabitables, qui n'appartiennent à personne, que l'on aborde difficilement, et d'où il semble impossible de sortir. Je m'arrêtai dans un certain amphithéâtre de rochers auquel pas une construction, pas un animal, pas une plante ne donnait de physionomie particulière. Il en avait une terrible, austère, désolée, qui n'appartenait à aucun pays, et qui pouvait ressembler à toute autre partie du monde qu'à l'Italie. (Lettre I ; p. 673 ; p. 62.)

Abstraite, géométrique, la montagne sert de décor universel à la solitude ; mais aussi d'instrument de mesure de la petitesse des hommes. Ainsi le mont Blanc, avec « ses grandes crêtes qui semblaient taillées dans les flancs de Paros, ses dents aiguës au pied desquelles nous étions comme des nains " (lettre $\mathrm{X}$; p. 9II ; p. 287).

\section{La pierre est dure}

George Sand ne s'en tient pas aux paysages mais cherche le contact avec le monde physique, celui de la minéralité, avec lequel elle développe des rap-

4. Sur ces questions, voir le très intéressant catalogue de l'exposition, Découverte et Sentiment de la montagne, 1740-1840 : à travers la collection Payot, Annecy, Conseil général de la Haute-Savoie, 1986.

5. Chateaubriand publie en 1806 un Voyage au Mont-Blanc, et Hugo un Fragment de voyage aux Alpes en I829, avant d'y revenir dans le Rhin de 1842. 
ports multiples. On trouve d'abord dans les Lettres d'un voyageur les représentations archétypales du monde de la roche, hostile au corps humain par ses dimensions, son aspect ou sa dureté.

La roche et la pierre font de la résistance à l'humain et marquent la limite de son pouvoir physique et moral : «[...] comme je marchais vite et regardant peu à terre, il m'arriva de me heurter contre une pierre et de tomber [...] » (lettre $V ;$ p. $774 ;$ p. I58), raconte le narrateur qui présente le désespoir comme " un obstacle semblable à une montagne écroulée " (lettre IV ; p. 75I ; p. 137). Tout chemin est bordé de pierres, qui symbolisent les dangers du voyage de la vie.

La pierre, qui cause la chute, peut aussi faire office de projectile. C'est l'instrument biblique traditionnel de la lapidation, au moins au sens figuré. " Tel je suis encore, $[\ldots]$ malgré tant de fange et de pierres qu'on m'a jetées [...] " (lettre IV; p. 743 ; p. I29), écrit le voyageur après son retour en France. Plus généralement elle est l'image de l'incontrôlé, du désordre, de la force qui va. Quatre ans après Hernani, en reprenant ce qui est déjà devenu un cliché du romantisme, George Sand peut écrire :

Comme une pierre détachée de la montagne, je roule au hasard, et les accidents du chemin décident seuls de mon impulsion. Cette pierre embarrassait les voies du destin, son souffle l'a balayée ; que lui importe où elle ira tomber? (Lettre I ; p. 657 ; p. 47.)

La force négative des " graviers stériles " et des " roches aiguës " est telle que le compagnon du Voyageur, ce jeune homme qui s'inquiétait « tant de la blancheur des marbres ", sombre dans un combat dément contre ces ennemis de pierre :

[...] tu poursuivais ta course, haletante parmi les rochers, tu les prenais corps à corps, tu luttais avec eux, et quand tu les avais renversés, tu partais avec un chant de triomphe, sans songer qu' ils t'encombraient dans leur chute et creusaient dans ton sein des blessures profondes ${ }^{6}$. (Lettre I ; p. $663 ;$ p. 53.)

Sand revient à plusieurs reprises sur le processus de "pétrification » qui saisit le vivant pour le réduire à l'insensibilité, puis à la mort. Regardant son visage pâle dans une source, le Voyageur immobile, gagné par le froid, s'effraie de son image en miroir : "Il me sembla que, moi aussi, je me pétrifiais » (lettre I ; p. 667 ; p. 57). Plus loin, après un autoportrait en paysan, il compare la rusticité que sa vie lui a léguée à la finesse de son ami musicien, Franz Liszt en l'espèce : "J'ai épaissi mon écorce, j’ai durci la peau de mes pieds sur les pierres de tous les chemins [...] Pourquoi suis-je devenu un

6. On peut voir dans ce corps à corps un souvenir du dénouement de Dom Juan. 
rocher? " (lettre VII ; p. 843 ; p. 223). Le réquisitoire contre Talleyrand qui occupe la lettre VIII en dresse ainsi le portrait : un cadavre à "face de marbre ", avec un caillou en guise de cœur. "On ouvrit une fenêtre : c'était celle du prince. - Depuis quand les cadavres ont-ils chaud ? dit mon ami en baissant la voix ; depuis quand les marbres ont-ils besoin de respirer l'air du soir ?" (p. $862 ;$ p. 24I). Plaisanterie méchante qui réunit délibérément la nécessité physique, la dureté morale et la métaphore minérale, mais qui dépasse radicalement le niveau des clichés de la langue courante.

Enfin, et l'on en revient au sculpteur, la lassitude peut conduire à l'échec, le feu sacré peut s'éteindre et l'art échouer à insuffler la vie à sa créature. "L'artiste est devenu Prométhée; mais l'homme d'argile s'est pétrifié et reste inerte sous le souffle divin " (lettre XI ; p. 93I-932 ; p. 308). Mais après tout qu'importe, si la statue de marbre est destinée à devenir poussière ?

Ces visions négatives peuvent se retourner : le caillou offre à la fois l'image de la dureté de cœur et celle de l'irréductibilité du juste, présenté comme " un caillou brut » (lettre IV ; p. 748 ; p. I34). La roche dure et inhumaine peut aussi se faire accueillante - et c'est là qu'on entre dans l'intime du monde sandien.

\section{La pierre est douce}

Outre le désir affirmé, face aux paysages de montagne les plus grandioses, de gravir les pics et de descendre par les brèches, le voyageur interrompt régulièrement sa marche pour des stations qui procèdent de la vraisemblance narrative et du souci d'introduire une description ou un dialogue. Il se retrouve alors dans une de ses positions favorites, " assis sur une roche " (lettre I), «sur une pierre au bord du chemin " (lettre V), sur un banc de pierre devant le château de Valençay (lettre VIII). C'est le lieu naturel et accueillant du repos et de la contemplation ; Canova lui-même est représenté assis sur une roche, contemplant comme le narrateur la campagne qui environne sa ville natale. $\grave{A}$ la différence du voyageur de Caspar David Friedrich, debout et immobile dans sa contemplation, celui de George Sand s'assied pour méditer sur le paysage : la différence de posture signale un rapport différent au monde. Audelà, ou plutôt en-deçà du visuel et du spirituel, s'instaure un contact physique, tactile avec le monde matériel.

La pierre offre le siège fonctionnel des temps rustiques, comme le trône d'Attila qu'on voit à Torcello, en fait une " chaise curule en pierre " qui servait aux préteurs romains pour percevoir l'impôt (lettre III). Siège assez confortable pour que le docteur, comparse du voyageur, puisse s'y endormir. 
De même à Venise, où l'on retrouve l'habitude populaire, décrite aussi dans Consuelo (chapitre III), de se coucher sur les dalles chaudes de soleil : " Pour dormir, il y a un endroit délicieux : c'est le perron de marbre blanc qui descend des jardins du vice-roi au canal " (lettre II ; p. 703 ; p. 90). Non seulement parce que le petit peuple vénitien n'a d' " autre oreiller qu'une marche de granit » (p. 704 ; p. 9I), mais parce que dormir ainsi, entre pierre et ciel, est un véritable plaisir que choisissent parfois des Vénitiens qui pourraient être installés plus confortablement chez eux. On songe à la pierre tombale qui, posée au-dessus du cercueil, abrite le sommeil définitif ; ici au contraire la pierre soutient le corps et sert de lit à un sommeil libre et heureux.

Elle peut également dresser un mur pour offrir sa protection : " Me voici au revers du rocher dans le plus profond du ravin. Je suis caché et abrité du vent comme dans une niche " (lettre IX; p. 876 ; p. 255). Ou, mieux encore, s'ouvrir en forme de grotte. On rencontre à plusieurs reprises ce motif de la grotte dans les Lettres d'un voyageur, toujours cachette et abri dans l'immensité de la nature - de même George Sand place fréquemment une petite chaumière dans les paysages qu' elle dessine - et jamais comme caverne, trou noir qui servirait de repaire à des bêtes, monstres et cyclopes homériques. La grotte d'Albert de Rudolstadt, dans Consuelo, offrira quelques années plus tard une autre version de cette maison maternelle et secrète, refuge inconnu des autres hommes, magnifié en château intérieur et lieu utopique de résurrection.

Pour le Voyageur, la grotte est lieu de régression et de plaisir physique. La première lettre évoque les trois grottes d'Oliero : l'une est un repaire de brigands, l'autre comporte un lac qui attire les touristes. La troisième, la grotte Parolini, possède une voûte de rochers, une source et un jardin naturel ; elle est couronnée par un rocher en forme de citadelle, "géant sublime " et " château magique "; c'est celle qu'élit le Voyageur. Et voici ce qu'écrit George Sand :

Contempler ce pic terrible, du fond de la grotte, au bord de la source, les pieds sur un tapis de violettes, entre la fraîcheur souterraine du rocher et l'air chaud du vallon, c'est un bien-être, c'est une joie que j'aurais voulu me retirer pour te l'envoyer. (P. 666-667 ; p. 56.)

Il est difficile de mieux dire le plaisir de la complémentarité du masculin et du féminin ; sans compter le fait que le « je " qui écrit est féminin, le destinataire masculin, et que cette velléité de générosité n'est pas dépourvue de perversité puisqu'elle est en réalité dans la grotte avec Pagello, ce que le destinataire, Musset, comme le lecteur, sont censés ignorer. Dans ces replis secrets de l'autobiographie s'inscrit en tout cas une véritable érotique de la grotte, représentation du sexe féminin, de l'origine du monde qu'un peu plus tard le 
peintre Gustave Courbet représentera obsessionnellement en peignant, dans son Jura natal, les grottes des sources de la Loue.

La grotte peut aussi devenir le lieu d'un autre acte d'amour, celui du cantique. "Gloire à Dieu dans les cieux et paix sur la terre aux hommes de bonnes intentions", chante le Voyageur sous l'écho de la voûte sonore, qui s'offre à la fois comme théâtre et sanctuaire naturel, à la fin de la lettre VIII. Le cantique vient récuser la vaine gloire du prince de Talleyrand, en opposant la conscience à la science ; la grotte abrite la communion de l'homme avec Dieu, l'infini de la prière musicale se déployant dans ce monde clos qui lui donne toute sa puissance. La nature sert alors de médiatrice entre l'humain et le divin.

La nature peut même procurer à l'humain l'illusion d'atteindre au divin. George Sand écrit : «[...] quand je me réveillai sur une cime des Alpes, il me sembla que, de mon pied, j'allais repousser la terre et m'élancer dans l'immensité " (lettre I ; p. 674; p. 63). Ce rêve d'un magistral coup de pied décide, dans sa puissance, de la plasticité du minéral : la montagne devient élastique et ploie sous l'élan, pour un vol triomphal qui évoque plus le char d'Apollon que la naïveté d'Icare. La roche permet à l'auteur tour à tour l'inscription secrète du féminin, maternel et érotique, et celle d'un fantasme masculin d'hyperpuissance : elle se prête comme une pâte au modelage de l'inconscient et au désir très fort de transcendance de la division sexuelle.

\section{L'eau et la pierre}

Sand connaissait mieux que personne la charge de merveilleux que recèlent les pierres, traces sur la terre des dieux ou des esprits, susceptibles de prendre regard et vie dans l'ombre menaçante et détentrices d'une énergie mystérieuse. Les Légendes rustiques peupleront le Berry de blocs de granit erratiques, mégalithes laissés par quelque déluge, et d'hommes de pierre pas tout à fait immobiles au bord des eaux de la Creuse ${ }^{7}$.

Pierres et rivières sont toujours inséparables, dans le temps par l'histoire géologique, dans l'espace par la genèse des paysages : «Au milieu de ce jardin immense, la Brenta coulait rapide et silencieuse sur un lit de sable, entre ces deux larges rives de cailloux et de débris de roches qu'elle arrache du sein des Alpes [...] » (lettre I ; p. 653 ; p. 43-44). Et donc par imbrication - la source

7. En particulier Les Pierres-Sottes ou Pierres-Caillasses. Voir Promenade dans le Berry, édition de G. Lubin, Bruxelles, Éditions Complexe, 1992. 
ou la rivière dans la grotte de pierre, les cailloux dans le lit de la rivière. Au terme de l'histoire de l'érosion agressive, les pierres dans l'eau et l'eau dans les pierres forment une union harmonieuse, qui vient rejoindre celle de la terre et du ciel selon une cosmogonie différente de celle de la Genèse où l'Éternel répartit le ciel, la terre et les eaux. Observons notre voyageur dans un moment de bonheur intense :

Me voici au revers du rocher dans le plus profond du ravin. Je suis caché et abrité du vent comme dans une niche. Le soleil réchauffe mes pieds mouillés dans l'herbe. Je les ai posés nus sur la pierre tiède et saine [...]. (Lettre IX ; p. 876 ; p. 255.)

Le rocher exclut le vent, accueille le soleil et redonne littéralement vie par la chaleur, tandis que l'eau et l'herbe sont froides. Les propriétés du minéral et de l'organique se trouvent pratiquement inversées.

Ailleurs le voyageur écoute " des gouttes de pluie qui se détachaient des branches et tombaient sur les rochers avec un petit bruit qui ressemblait à celui d'un baiser" (lettre I ; p. 660 ; p. 50). Tendresse insolite, qui permet de comprendre que Sand n'a aimé qu'une ville, Venise, la seule à lui offrir ce mariage d'amour de la pierre et de l'eau.

Cette vision affective, voire irénique de l'ordre du monde repose en réalité sur une exigence de connaissance. Voir et admirer sans comprendre est parfaitement vain selon le major, qui se moque du Voyageur et l'accuse de s'en tenir aux apparences, ce qui n'avance à rien : « [...] vous pourriez tracer dans votre cerveau une ligne exacte des déchiquetures de la chaîne $[. .$.$] » (lettre X;$ p. $906 ;$ p. 283). Tandis que le Voyageur réduit le monde à un assemblage de lignes et de couleurs, lui recherche très sérieusement le principe de l'univers. «Un rocher de granit ne ressemble pas plus à un bloc de grès pour le ton et l'attitude, pour le caractère, et pour l'impression qu'on en reçoit qu'un More ne ressemble à un Anglais ${ }^{8}$ ", écrit George Sand à un peintre paysagiste de ses amis. Dans les années I860, elle se montre " enragée » de géologie et de minéralogie, collectionnant et classifiant les pierres, comme d'ailleurs les plantes et les coquillages, passionnée à la fois par les sciences de la terre et les sciences du vivant.

Dans Laura, voyage dans le cristal, roman de I863, le narrateur, observant pour la première fois une géode à la loupe, raconte :

J'y vis des particularités de forme et de couleur qui, agrandies par l'imagination, composaient des sites alpestres, de profonds ravins, des montagnes grandioses, des glaciers, tout ce qui constitue un tableau imposant et sublime dans la nature?

8. G. Sand, lettre à Eugène Fromentin du 27 mars 1857, Correspondance, op. cit., vol. XIV, p. 289. La graphie " More " est de Sand.

9. G. Sand, Laura, voyage dans le cristal (I865), Toulouse, Éditions Ombres, I993, p. II-I2. 
Elle voit cette nature qui « travaille mieux que les fées » de la même manière que le géologue et poète Novalis. Le vieux mineur qui apparaît dans Heinrich von Ofterdingen décrit ainsi ses voyages géologiques et l'exploration des richesses de la terre :

Ce que je voyais était du métal le plus précieux et ciselé à ravir. Dans les boucles précieuses et les ramures de l'argent pendaient, brillants, transparents, des fruits de rubis et ces arbustes comblés jaillissaient d'un sol de cristal d'un travail absolument inimitable ${ }^{\mathrm{ro}}$.

Ce qui pose directement la question de l'art :

Quant aux tableaux, la Nature n'est-elle pas la sublime maîtresse ? Elle crée d'innombrables figures, belles et singulières, dispose les lumières et les ombres de telle sorte qu'une main exercée, un œil juste, la science de la préparation et du mélange des couleurs, permettent son imitation parfaite ${ }^{\mathrm{II}}$.

L'art ne peut naître, pour Novalis comme pour Sand, que de la réconciliation de l'homme avec la nature.

Déjà Jules Néraud, le Malgache dont le Voyageur raconte la biographie dans la lettre VI et qui partage avec l'auteur un " amour à la fois immense et minutieux de la nature » (p. 805 ; p. I86), offre à la femme qu'il aime ce qui est pour lui le plus beau des cadeaux, " un morceau de cristal de Madagascar, un fragment de basalte de la montagne du Pouce» (p. 803 ; p. 185) qu'elle conserve précieusement sous verre.

Les seules sculptures qui vaillent, en définitive, sont œuvre divine :

Au-dessus des feux, au-dessus de la fumée et de la brume, la chaîne du MontBlanc montrait une de ces dernières ceintures granitiques, noire comme l'encre et couronnée de neige. Ces plans fantastiques du tableau semblaient nager dans le vide. Sur quelques cimes que le vent avait balayées, apparaissaient, dans un firmament pur et froid, de larges étoiles. Ces pics de montagnes, élevant dans l'éther un horizon noir et resserré, faisaient paraître les astres étincelants. (Lettre X; p. 902 ; p. 279.)

Une étoile de feu, une autre bleue comme une larme viennent couronner cette belle description qui est le fait d'une autre œuvre artistique : l'œuvre littéraire. Ce n'est pas au profit de la nature que l'art a été éliminé, mais au profit de la littérature : c'est à elle que Sand a donné le pouvoir.

IO. Novalis, Heinrich von Ofterdingen (I80I), in Romantiques allemands, édition de M. Alexandre, Paris, Gallimard "Bibliothèque de la Pléiade », I966, t. I, p. 446.

II. Ibid., p. 397. 
Dans le débat, récurrent dans tout le premier tiers du XIXe siècle, sur la nature et l'idéal, débat que le romantisme aurait à la fois repris et dramatisé d'après Paul Bénichou ${ }^{12}$, une question reste sans réponse, celle de l'origine du Beau : modèle d'origine divine, ou construction de l'esprit humain ? De ce côté, la réponse sandienne est claire : les productions humaines sont inférieures aux beautés de la nature ; l'homme peut les adorer, et tâcher de les connaître. Mais ranger les Lettres d'un voyageur dans la catégorie d'un naturalisme spiritualiste présente un intérêt limité.

Ce qui est plus intéressant, dans ces années I830 où prévaut la religion de l'art, c'est ce détournement de l'art opéré par Sand au profit de la littérature. C'est le discours de l'écrivain qui délite les marbres du sculpteur, qui déclare le beau, le divin, la montagne ; qui construit un rapport ambivalent entre l'homme et le monde, retournant les forces négatives en forces bienfaisantes par l'investissement du fantasme, conjugué à l'exercice de la pensée et de la connaissance ; qui se construit enfin lui-même dans une forme fragmentaire dont le choix de l'épistolaire ne suffit pas à rendre compte.

George Sand affirme avec force la puissance des mots, non pas pour réhabiliter les vieilles hiérarchies de l'ut pictura poesis, mais parce que la poésie est seule à pouvoir parler de tout le reste - à pouvoir parler. C'est en ce sens que l'on peut évoquer une poétique des Lettres d'un voyageur, comme mise en question et en exercice des pouvoirs du langage.

C'est ainsi que Solange, la petite fille, promet à sa mère : "Sois tranquille, mon George ; quand je serai reine, je te donnerai tout le mont Blanc » (lettre X; p. $903 ;$ p. 280). 
I2. P. Bénichou, Le Sacre de l'écrivain, I750-1830, Paris, Gallimard, I996 (rééd.), p. 225-226. 